\title{
Polishing of optical media by dielectric barrier discharge inert gas plasma at atmospheric pressure
}

\author{
C. Gerhard \\ gerhard@hawk-hhg.de
}

T. Weihs

A. Luca

S. Wieneke

W. Viöl

\begin{abstract}
University of Applied Sciences and Arts, Laboratory of Laser and Plasma Technologies, Von-OssietzkyStraße 99, 37085 Göttingen, Germany

Fraunhofer Institute for Surface Engineering and Thin Films, Application Center for Plasma and Photonics, Von-Ossietzky-Straße 99, 37085 Göttingen, Germany

University of Applied Sciences and Arts, Laboratory of Laser and Plasma Technologies, Von-OssietzkyStraße 99, 37085 Göttingen, Germany

Roth \& Rau AG, An der Baumschule 6-8, 09337 Hohenstein-Ernstthal, Germany
\end{abstract}

University of Applied Sciences and Arts, Laboratory of Laser and Plasma Technologies, Von-OssietzkyStraße 99, 37085 Göttingen, Germany

Fraunhofer Institute for Surface Engineering and Thin Films, Application Center for Plasma and Photonics, Von-Ossietzky-Straße 99, 37085 Göttingen, Germany

University of Applied Sciences and Arts, Laboratory of Laser and Plasma Technologies, Von-OssietzkyStraße 99, 37085 Göttingen, Germany

Fraunhofer Institute for Surface Engineering and Thin Films, Application Center for Plasma and Photonics, Von-Ossietzky-Straße 99, 37085 Göttingen, Germany

In this paper, surface smoothing of optical glasses, glass ceramic and sapphire using a low-power dielectric barrier discharge inert gas plasma at atmospheric pressure is presented. For this low temperature treatment method, no vacuum devices or chemicals are required. It is shown that by such plasma treatment the micro roughness and waviness of the investigated polished surfaces were significantly decreased, resulting in a decrease in surface scattering. Further, plasma polishing of lapped fused silica is introduced. Based on simulation results, a plasma physical process is suggested to be the underlying mechanism for initialising the observed smoothing effect. [DOI: http://dx.doi.org/10.2971/jeos.2013.13081]

Keywords: Atmospheric pressure plasma, polishing, optical glasses, glass ceramic, sapphire, scattering

\section{INTRODUCTION}

For a number of high-end systems and devices such as laser sources, UV lithography optics and high performance mirrors, precisely-shaped and -polished optics surfaces are required. Against this background, precision polishing of relevant optical media is of great interest in order to realise surface roughnesses in the nanometre or even angstrom range. Such precision smoothing can be achieved by classical polishing using pitch tools and a polishing agent, i.e. a suspension of water and fine abrasives [1]. However, this technique is usually limited to spherical and plane surfaces. Polishing of glass surfaces is also achieved by laser-induced heating above the glass transition temperature. As a result of the accompanying decrease in viscosity, material flow due to surface tension mechanisms occurs [2]. Here, carbon dioxide $\left(\mathrm{CO}_{2}\right)$ lasers are usually employed due to the improved energy coupling at glass surfaces at this particular laser wavelength of $10.6 \mu \mathrm{m}$. Beyond laser processing, atmospheric pressure plasmas (APP) are suitable for thermally-induced polishing of rough glass surfaces. Paetzelt et al. reported such polishing of fine ground fused silica by a microwave-powered APP jet source where a mixture of argon and helium was used as process gas. Applying this tech- nique at a microwave power of $135 \mathrm{~W}$, the surface arithmetic mean roughness $R a$ was significantly decreased from $550 \mathrm{~nm}$ to $0.64 \mathrm{~nm}$ due to a surface heating to $1900 \mathrm{~K}$ [3]. Polishing of glasses and silicon (Si) -based media in general at low temperature can be performed by APP techniques using fluorine (F) -containing process gasses. Here, material removal is achieved by chemical reactions according to

$$
\mathrm{SiO}_{2}+4 \mathrm{~F} \rightarrow \mathrm{SiF}_{4}+\mathrm{O}_{2}
$$

and

$$
\mathrm{Si}+4 \mathrm{~F} \rightarrow \mathrm{SiF}_{4}
$$

respectively. Applying this technique, Zhang et al. accomplished smoothing of silicon surfaces where a reduction from 2.39 to $1 \mathrm{~nm}$ (root mean squared roughness $R q$ ) and 1.76 to $0.63 \mathrm{~nm}(R a)$ was achieved [4]. A reduction in surface roughness was also reported in the case of plasma jet machining of silicon carbide ( $\mathrm{SiC}$ ) surfaces [5]. Yao et al. and Jin et al. presented plasma machining of Zerodur ${ }^{\circledR}$ using a RF-excited plasma jet at atmospheric pressure. It was shown that the surface roughness was strongly dependent on the process parameters and in particular on the mixing ratio of the working gas, 


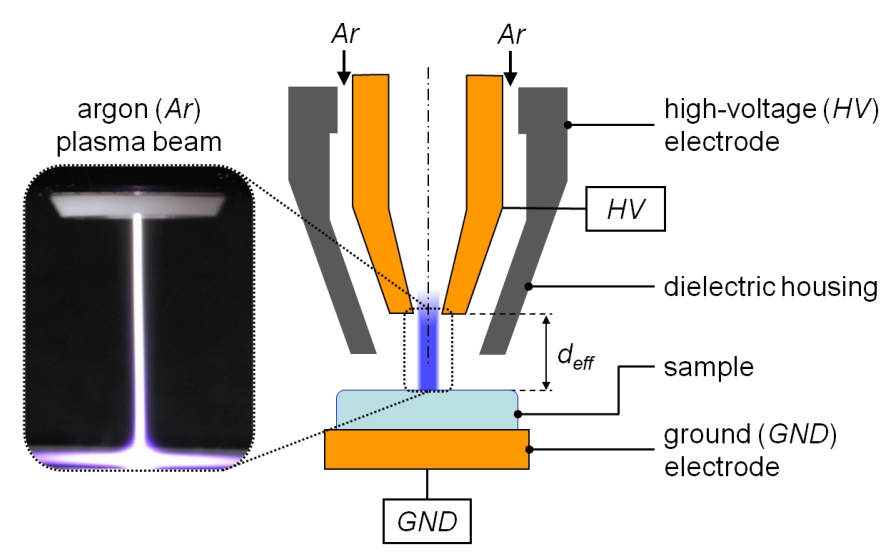

FIC. 1 Experimental setup for APP polishing of optical media.

$\mathrm{SF}_{6}$ and $\mathrm{O}_{2}[6,7]$. However, such plasma figuring methods can provoke surface roughening due to inhomogeneous etching, an effect which was also observed in the case of reactive ion etching (RIE) of pyrex glass [8]. Such roughening can be overcome by the choice of the applied gas mixture in order to mitigate the sputter effect of heavy ions from the inert gases [9].

Against this background, atmospheric pressure plasma polishing of different technically relevant optical media is presented in this contribution. Here, the goal was not to perform any surface figuring but to provide a novel technique for surface smoothing based on a chemically neutral APP. In contrast to existing APP techniques, surface smoothing was achieved at low temperature below $100^{\circ} \mathrm{C}$ and low power without applying any reactive process gas mixtures.

\section{EXPERIMENTAL SETUP AND PROCEDURE}

Plasma polishing experiments were performed on optically polished plane samples made of fused silica Suprasil 3, boron crown glass BK7, glass ceramic Zerodur ${ }^{\circledR}$ and sapphire. These materials are well-established and commonly used for the realisation of high precision optical devices as for example, in case of fused silica and sapphire, UV optics. In this context, precision polishing and finishing in the (sub)nanometer scale is of great interest in order to reduce scattering and interreflection effects. For plasma polishing of these optical media, a dielectric barrier discharge (DBD) at atmospheric pressure was applied using a rotational-symmetric cone-shaped plasma source [10, 11]. As shown in Figure 1, this plasma source consists of an internal high-voltage (HV) electrode and an external ground (GND) electrode. The dielectric separation of both electrodes was realised by the particular sample itself, where the effective discharge gap $d_{\text {eff }}$ was $21 \mathrm{~mm}$.

By the process gas flow, i.e. argon (Ar) 4.6 from Linde at a flow rate of 4 standard litres per minute ( $\mathrm{slm}$ ), a stable compressed plasma beam with an $1 / \mathrm{e}^{2}$ diameter of approx. $200 \mu \mathrm{m}$ was formed within the discharge gap (compare inset in Figure 1). However, the resulting plasma tracing point on the sample surface showed a surface discharge-like behaviour due to an accumulation of charge carriers. This tracing point was several millimetres in diameter, featuring a Gaussian rotation

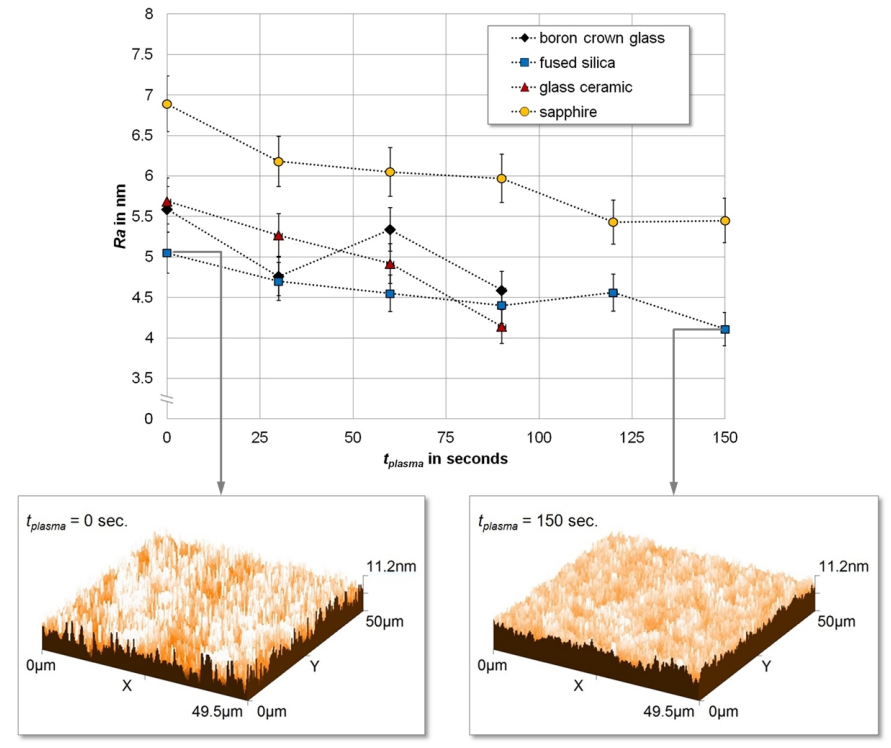

FIG. 2 Arithmetic mean roughness $R a$ of different optical media vs. plasma treatment duration $t_{\text {plasma }}$ including $3 \mathrm{D}$ AFM views of polished fused silica at $t_{\text {plasma }}=0$ (left) and 150 (right) seconds.

symmetric intensity distribution as confirmed by high-speed camera measurements. During the experiments, neither the plasma source nor the sample was moved. The plasma source was driven at a pulse repetition rate frep of $7 \mathrm{kHz}$ with an averaged plasma power $P_{a v}$ of $1.19 \mathrm{~W}$. Taking the applied plasma energy of $0.17 \mathrm{~mJ}$ for each HV-pulse train with a duration of approx. $80 \mu$ s into account, the fluence per pulse within the plasma beam can be calculated to amount to $540 \mathrm{~mJ} / \mathrm{cm}^{2}$. As ascertained by spectroscopic measurements, the electron temperature was approx. $1 \mathrm{eV}$. Plasma treatment was performed in steps of 30 seconds up to a total treatment time of 90 seconds (boron crown glass, glass ceramic) and $150 \mathrm{sec}-$ onds (fused silica, sapphire), respectively. For each step, both the arithmetic mean roughness $R a$ and the root mean squared roughness $R q$ were measured by the use of an atomic force microscope (AFM) easyScan 2 AFM from Nanosurf where the measured area was $50 \times 49.5 \mu \mathrm{m}^{2}$.

For comparison, plasma polishing using argon as process gas (flow rate $=0.9 \mathrm{slm}$ ) was also performed on lapped fused silica surfaces. Here, the plasma beam diameter was approx. $500 \mu \mathrm{m}$ whereas the effective discharge gap amounted to $14.5 \mathrm{~mm}$. The fluence per pulse was $15 \mathrm{~mJ} / \mathrm{cm}^{2}$. Plasma treatment was carried out in steps of 10 minutes up to a total treatment time of 60 minutes. In addition to the roughness parameters $R a$ and $R q$, the transmission $T$ was measured after each step using an UV/VIS-spectrometer Lambda 650 from Perkin Elmer.

\section{RESULTS}

By the plasma treatment as described above, significant topographic surface modifications of the investigated optical media were achieved. As shown in Figure 2 and listed in Table 1, $R a$ was continuously decreased with increasing plasma treatment duration. The same effect was observed for $R q$. 


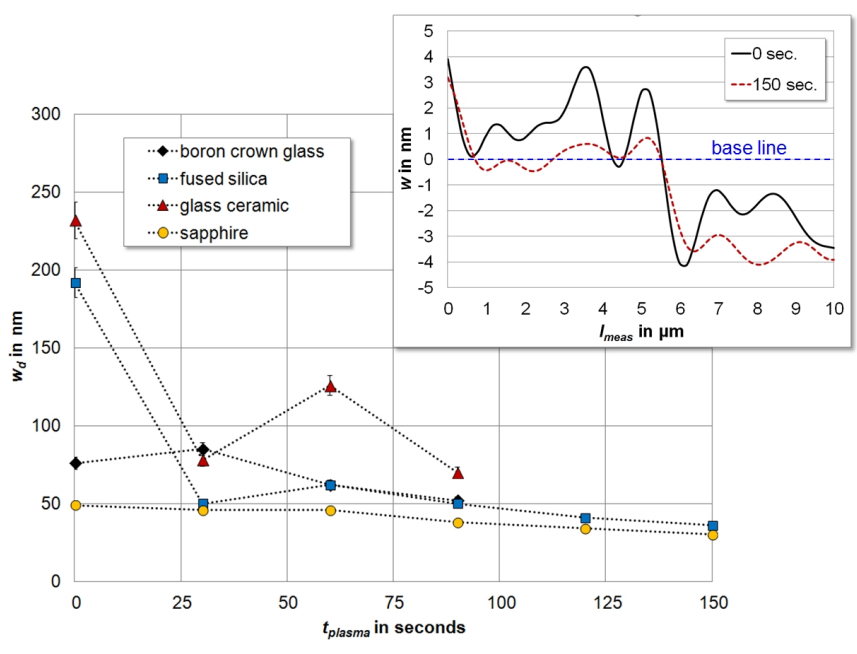

FIG. 3 Maximum depth of waviness $w_{d}$ of different optical media vs. plasma treatment duration $t_{\text {plasma }}$, inset: example for waviness $w$ of polished fused silica at $t_{\text {plasma }}=0$ and 150 seconds vs. measuring length $I_{\text {meas }}$.

Such surface smoothing of optical glasses was already observed in previous work where the same plasma source and treatment procedure was applied to barite crown glass $\mathrm{N}$ BaK4 and heavy flint glass SF5. After a plasma treatment duration of 60 seconds, $R a$ was reduced by $7.8 \%$ (N-BaK4) and $59.7 \%$ (SF5), respectively [12]. Generally, a reduction in surface roughness comes along with a decrease of the surface contact angle, corresponding to an increase in total surface energy $\gamma_{s}$ [13]. Against this background, $\gamma_{s}$ was measured using a Contact Angle Measurement System G10 from Krüss. For instance, $\gamma_{s}$ of fused silica was increased from $68.66 \mathrm{~mJ} / \mathrm{m}^{2}$ to $72.69 \mathrm{~mJ} / \mathrm{m}^{2}$ (i.e. $+5.9 \%$ ) after a plasma treatment duration of 60 seconds, verifying the observed surface smoothing at this instant of time $(\Delta R a=-9.9 \%$ and $\Delta R q=-7.5 \%)$. Moreover, no mentionable change in the polar fraction of the total surface energy was detected. Since this parameter is directly related to the molecular structure and orientation of near-surface bonds within the sample bulk material it can thus be stated that no modification in chemical and, as a consequence, optical properties occurred due to the plasma treatment.

In addition to the roughness parameters, the waviness $w$ as well as the maximum depth of waviness $w_{d}$ was extracted from the AFM profiles using the evaluation software Gwyddion from the Department of Nanometrology of the Czech Metrology Institute. Here, the measuring length $l_{\text {meas }}$ was $70 \mu \mathrm{m}$, i.e. the diagonal of the overall measured area. As also observed in the case of roughness parameters, the depth of waviness of the investigated optical media was reduced continuously by the plasma treatment as shown in Figure 3.

According to DIN 4760, the surface roughness is a $3^{\text {rd }}$ and $4^{\text {th }}$ order irregularity of form whereas the waviness represents a $2^{\text {nd }}$ order form deviation. It can thus be stated that as a result of the plasma treatment not only high order but also low order irregularities were decreased as listed in Table 1.

For comparison, plasma polishing was also carried out on lapped fused silica surfaces with a comparatively high initial surface roughness. As shown in Figure 4, a considerable

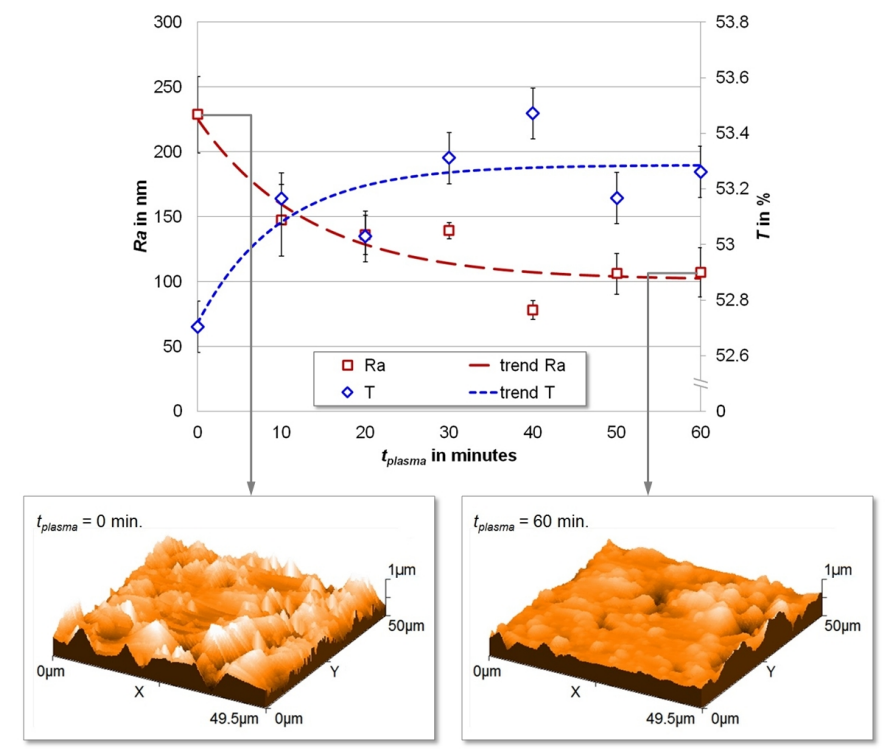

FIG. 4 Comparison of the arithmetic mean roughness $R a$ and the transmission $T$ $(\lambda=193 \mathrm{~nm})$ of a lapped fused silica sample vs. plasma treatment duration $t_{\text {plasma }}$ including 3D AFM views of lapped fused silica at $t_{\text {plasma }}=0$ (left) and 60 (right) minutes.

\begin{tabular}{|l|c|c|}
\hline & $\begin{array}{c}\Delta R a \\
(\%)\end{array}$ & $\begin{array}{c}\Delta w_{d} \\
(\%)\end{array}$ \\
\hline boron crown glass & -17.9 & -31.6 \\
fused silica & -18.6 & -81.3 \\
glass ceramic & -27.2 & -69.8 \\
sapphire & -20.9 & -38.8 \\
\hline
\end{tabular}

TABLE 1 Absolute change $\Delta$ in arithmetic mean roughness $R a$ and maximum depth of waviness $w_{d}$ after particular overall plasma treatment duration.

decrease in surface roughness was achieved by such plasma treatment. Consequently, the transmission $T$ was progressively increased.

The increase in transmission as a result of the reduction of diffuse reflection on the rough fused silica surface is further supported by the measured decrease in waviness by a factor of 4.8 after a plasma treatment duration of 60 minutes. The observed decrease in both surface roughness and waviness of polished and lapped surfaces clearly indicates a selective material removal of roughness peaks and contour maxima by the plasma treatment.

\section{DISCUSSION}

In previous work, the heating of a glass sample surface exposed to the used plasma beam was measured using an infrared camera VarioCAM ${ }^{\circledR}$ from InfraTec/Jenoptik. Here, a surface temperature of $88^{\circ} \mathrm{C}$ was determined [14]. Since this temperature is significantly below the softening temperature of the investigated media, thermal smoothing due to surface melting (which is the underlying mechanism in the case of laser-induced surface smoothing) can be excluded in the present case. The presented method thus differs significantly from thermal APP jet polishing as reported by Paetzelt et al. [3] (compare introduction section). The 
observed decrease in surface roughness and waviness is rather explained by plasma physical effects. In principle, the concentration of argon plays an important role for plasma etch mechanisms. Li et al. showed that during etching of fused silica by the use of fluorine-based plasmas, an increase in argon concentration allows increasing the etch rate [15]. For pure silicon, Coburn and Winters observed certain etching by pure argon ion bombardment [16]. Against this background, the presented surface smoothing using argon as inert process gas can be explained by different mechanisms: First, a slight argon ion bombardment by reason of the formation of plasma sheath at the sample surface, resulting in an acceleration of ions towards the surface [17], can contribute to material removal. Second, material removal can be due to the deexcitation of excited argon and metastable argon species and an accompanying energy transfer to the sample surface. Here, the possible underlying effects are electron quenching, twoand three-body collisions with argon atoms and Penning ionisation at the sample surface [12]. The existence of such species in the near-surface area can most likely be assumed due the direct plasma ignition on the substrate based on electron excitation and ion-impact excitation in the plasma sheath close to the sample surface [18] and/or resonant neutralisation. In the last case, argon metastables are provided as a result of the recombination of argon ions with the negative surface charge on the dielectric sample [19]. However, both effects, ion bombardment and de-excitation of argon species, should result in an almost uniform material removal. Due to the selective removal of surface texture maxima it can thus be assumed that the plasma discharge causes high electric field strengths at roughness peaks, initialising the observed erosion effect. This assumption was confirmed by a simulation of the distribution of the electric field strength on a rough dielectric surface within a parallel-plate capacitor, which is an appropriate model for the plasma discharge used in the present work. Such simulation was performed using the COMSOL Multiphysics software. The simulated dielectric was fused silica with different micro volume elements (i.e. hemispheres with radii $r$ in the range from 1 to $100 \mathrm{~nm}$ and a pyramidal peak with a height $h$ of $150 \mathrm{~nm}$ and a point angle $\alpha$ of $30^{\circ}$ ), representing the roughness and waviness profile. As shown in Figure 5, the highest electric field strengths are found on the top of the particular volume elements whereas low electric field strengths occur within the valleys in between roughness peaks and surface texture maxima, respectively.

This simulated behaviour confirms a selective material removal of maxima at rough and wavy surfaces. An increase in electric field strength due to an increased electrode surface roughness, initialising an additional potential, was also already reported by Zhao et al. on the example of a parallelplate capacitor [20]. The above-presented surface smoothing of polished optical media has a considerable impact on surface scatter characteristics. Basically, the amount of light scattered at a technical surface can be calculated from its root mean squared roughness $R q$ by applying the total integrated scatter (TIS) which is given by

$$
\text { TIS }=1-e^{-\left(\frac{4 \pi \cdot R q \cdot \cos A O I}{\lambda}\right)^{2}}
$$

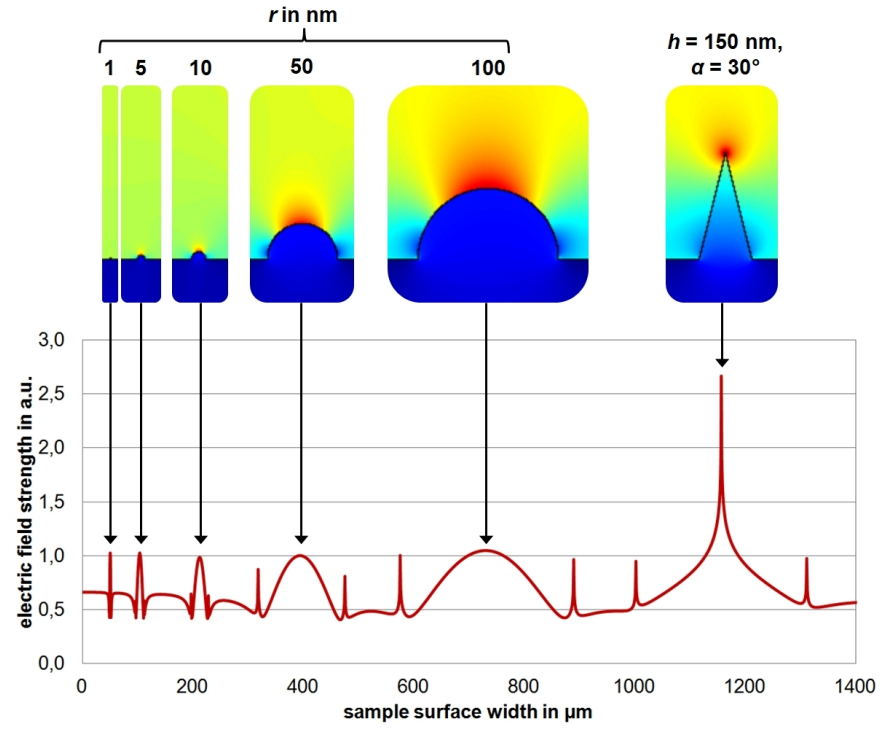

FIG. 5 Qualitative distribution of the electric field strength on a dielectric surface (bottom) with micro volume elements (top).

Here, $A O I$ is the angle of incidence of the incoming light and $\lambda$ its wavelength [21]. As an example, at perpendicular incidence $(A O I=0)$ and a wavelength of $546 \mathrm{~nm}$, TIS was reduced by a factor of 1.08 (boron crown glass), 1.17 (fused silica), 1.62 (glass ceramic) and 1.38 (sapphire), respectively, after a plasma treatment duration of 60 seconds. Regarding the quality of imaging optics, such decrease in surface scattering generally improves the contrast ratio. The observed decrease in waviness further contributes to a reduction of blurring. As a result of these plasma-induced surface effects, the modulation transfer function (MTF) of an optical system could be improved by the presented method. Moreover, image distortions could be mitigated in consequence of the diminution of form deviations.

\section{CONCLUSIONS}

The presented low-power plasma polishing technique allows a considerable surface smoothing of different optical media of high technical relevance. In the case of polished surfaces, a reduction in surface roughness by approx. 18-28\% was achieved. In contrast to other precision polishing techniques using lasers or hot plasmas, surface smoothing is not induced by heating. Thermally-induced disturbing effects such as stress birefringence are thus avoided. The presented method could be applied for post-processing and finishing of high-end optics after precision figuring, for example by RIE or ion beam etching (IBE) methods in order to reduce surface scattering. However, relatively high final roughness values were achieved in comparison to other techniques which are based on thermally-induced mechanisms. Further, the observed removal of roughness peaks approaches a certain saturation which can be explained by the smoothing process and the accompanying increasing uniformity of the electric field strength distribution. In ongoing work, the variation of crucial plasma parameters will thus be investigated in order to improve the process efficiency and to evaluate the limits of this method. Here, a continuous and regulated increase in 
voltage during the plasma polishing process is of essential interest to maintain the required high electric field strengths on the top of the continuously shrinking roughness peaks and waviness maxima. Owing to the advantage of a negligible substrate heating due to the used dielectric barrier discharge, which is commonly referred to as a cold plasma, the treatment of temperature-sensitive optical media and materials such as coatings is made possible by the presented method. The smoothing of such surfaces will be investigated in the near future.

\section{ACKN OWLEDGEMENTS}

This work was supported by the European Regional Development Funds (EFRE) and the Workgroup Innovative Projects of Lower Saxony (AGiP) in the frame of the Lower Saxony Innovation Network for Plasma Technology (NIP), project funding reference number W2-80029388.

\section{References}

[1] L. M. Cook, "Chemical processes in glass polishing," J. Non-Cryst. Solids 120, 152-171 (1990).

[2] C. Buerhop, B. Blumenthal, R. Weissmann, N. Lutz, and S. Biermann, "Glass surface treatment with excimer and $\mathrm{CO}_{2}$ lasers," Appl. Surf. Sci. 46, 430-434 (1990).

[3] H. Paetzelt, G. Böhm, and T. Arnold, "Plasma jet polishing of rough fused silica surfaces," in Proceedings to euspen $13^{\text {th }}$ International Conference, 19-22 (euspen, Berlin, 2013).

[4] J. Zhang, B. Wang, and S. Dong, "Application of atmospheric pressure plasma polishing method in machining of silicon ultrasmooth surfaces," Front. Electr. Electron. Eng. China 3, 480-487 (2008).

[5] T. Arnold, G. Boehm, I.-M. Eichentopf, M. Janietz, J. Meister, and A. Schindler, "Plasma Jet Machining - A novel technology for precision machining of optical elements," Vakuum in Forschung und Praxis 22, 10-16 (2010), in German.

[6] Y. X. Yao, B. Wang, J. H. Wang, H. L. Jin, Y. F. Zhang, and S. Dong, "Chemical machining of Zerodur material with atmospheric pressure plasma jet," CIRP Ann-Manuf. Techn. 59, 337-340 (2010).

[7] H. L. Jin, B. Wang, and F. H. Zhang, "Effect on surface roughness of zerodur material in atmospheric pressure plasma jet processing," in Proc. SPIE 7655, 76552X (2010).

[8] X. Li, T. Abe, and M. Esashi, "Deep reactive ion etching of Pyrex glass using SF$_{6}$ plasma," Sensor. Actuator. A 87, 139-145 (2001).
[9] L. Li, T. Abe, and M. Esashi, "Smooth surface glass etching by deep reactive ion etching with $\mathrm{SF}_{6}$ and Xe gases," J. Vac. Sci. Technol. B 21, 2545-2549 (2003).

[10] S. Brückner, S. Rösner, C. Gerhard, S. Wieneke, and W. Viöl, "Plasma-based ionisation spectroscopy for material analysis," Mater. Test. 53, 639-642 (2011).

[11] W. Viöl, S. Wieneke, S. Brückner, and R. Damm, "Hollow funnelshaped plasma generator" Patent Application WO 2011/095245 (2011).

[12] C. Gerhard, S. Roux, S. Brückner, S. Wieneke, and W. Viöl, “Lowtemperature atmospheric pressure argon plasma treatment and hybrid laser-plasma ablation of barite crown and heavy flint glass," Appl. Optics 51, 3847-3852 (2012).

[13] J. Lawrence, and L. Li, "Wettability characteristics of an $\mathrm{Al}_{203} / \mathrm{SiO}_{2}$ based ceramic modified with $\mathrm{CO}_{2}, \mathrm{Nd}: \mathrm{YAG}$, excimer and highpower diode lasers," J. Phys. D Appl. Phys. 32, 1075-1082 (1999).

[14] A. Gredner, C. Gerhard, S. Wieneke, K. Schmidt, and W. Viöl, "Increase in generation of poly-crystalline silicon by atmospheric pressure plasma-assisted excimer laser annealing," J. Mater. Sci. Eng. B 3, 346-351 (2013).

[15] X. Li, L. Ling, X. Hua, M. Fukasawa, and G.S. Oehrlein, "Effects of $\mathrm{Ar}$ and $\mathrm{O}_{2}$ additives on $\mathrm{SiO}_{2}$ etching in $\mathrm{C}_{4} \mathrm{~F}_{8}$-based plasmas," J. Vac. Sci. Technol. A 21, 284-293 (2003).

[16] J. W. Coburn, and H. F. Winters, "Ion- and electron-assisted gassurface chemistry-An important effect in plasma etching," J. Appl. Phys. 50, 3189-3196 (1979).

[17] I. Langmuir, "Positive ion currents from the positive column of mercury arcs," Science 58, 290-291 (1923).

[18] N. Baguer, A. Bogaerts, Z. Donko, R. Gijbels, and N. Sadeghi, "Study of the Ar metastable atom population in a hollow cathode discharge by means of a hybrid model and spectrometric measurements," J. Appl. Phys. 97, 123305 (2005).

[19] B. Niermann, R. Reuter, T. Kuschel, J. Benedikt, M. Böke, and J. Winter, "Argon metastable dynamics in a filamentary jet microdischarge at atmospheric pressure," Plasma Sources Sci. Technol. 21, 034002 (2012).

[20] Y.-P. Zhao, G.-C. Wang, and T.-M. Lu, "Surface-roughness effect on capacitance and leakage current of an insulating film," Phys. Rev. B 60, 9157-9164 (1999).

[21] H. E. Bennett, and J. 0. Porteus, "Relation between surface roughness and specular reflectance at normal incidence," J. Opt. Soc. Am. 51, 123-129 (1961). 